\title{
Ultra-Low-Dose Naloxone Restores the Antinociceptive Effect of Morphine and Suppresses Spinal Neuroinflammation in PTX-Treated Rats
}

\author{
Ru-Yin Tsai', Fong-Lin Jang ${ }^{2}$, Yueh-Hua Tai', Shinn-Long Lin ${ }^{1,3}$, Ching-Hui Shen ${ }^{1,4}$ and Chih-Shung Wong*,3 \\ 'Graduate Institute of Medical Science, National Defense Medical Center, Taipei, Taiwan; ${ }^{2}$ Department of Psychiatry, Chi-Mei Medical Center, \\ Yong-Kong, Tainan, Taiwan; ${ }^{3}$ Department of Anesthesiology, Tri-Service General Hospital, National Defense Medical Center, Taipei, Taiwan; \\ ${ }^{4}$ Department of Anesthesiology, Taichung Veterans General Hospital, Taipei, Taiwan
}

\begin{abstract}
The aim of the present study was to examine the effect of ultra-low-dose naloxone on pertussis toxin (PTX)-induced thermal hyperalgesia in rats and its underlying mechanisms. Male Wistar rats, implanted with an intrathecal catheter with or without a microdialysis probe, received a single intrathecal injection of PTX ( I $\mu \mathrm{g}$ in $5 \mu$ l saline). Four days after PTX injection, they were randomly given a different dose of naloxone (either $15 \mu \mathrm{g}$ or $15 \mathrm{ng}$ in $5 \mu \mathrm{l}$ saline), followed by a morphine injection ( $10 \mu \mathrm{g}$ in $5 \mu \mathrm{l}$ saline) after $30 \mathrm{~min}$. The results found that PTX injection induced thermal hyperalgesia and increasing excitatory amino acid (EAA; L-glutamate and L-aspartate) concentration in the spinal CSF dialysates. Ultra-low-dose naloxone not only preserved the antinociceptive effect of morphine but also suppressed the PTX-evoked EAA release as well. Moreover, ultra-low-dose naloxone plus morphine administration inhibited the downregulation of L-glutamate transporters (GTs) and the L-glutamate-metabolizing enzyme glutamine synthetase (GS), and, moreover, inhibited microglial activation and suppressed cytokine expression in PTX-treated rat spinal cords. These results show that ultra-low-dose naloxone preserves the antinociceptive effect of morphine in PTX-treated rats. The mechanisms include (a) inhibition of pro-inflammatory cytokine expression, (b) attenuation of PTX-evoked EAA release, and (c) reversion of the downregulation of GT expression.
\end{abstract}

Neuropsychopharmacology (2008) 33, 2772-2782; doi: I0.I 038/sj.npp. I 30 I672; published online 23 January 2008

Keywords: excitatory amino acid; IL- I $\beta$; IL-6; TNF- $\alpha$; opioid receptor; pro-inflammatory cytokine

\section{INTRODUCTION}

Opioids are the therapeutic mainstay for alleviation of acute and chronic pains. However, studies have shown that morphine is less effective in treating neuropathic pain. Gilron et al (2005) have demonstrated that the maximal tolerated dose of morphine produced only a $26-28 \%$ reduction in neuropathic pain, owing to incomplete efficacy and/or dose-limiting adverse effects. A combination of mechanistically distinct analgesics may provide either an additive or synergistic effect to improve the efficacy of morphine at lower dose with fewer side effects than the use of a single agent or a high dose of morphine.

Neuropathic pain is initiated or caused by a primary lesion or dysfunction in the nervous system. The underlying cellular and molecular mechanisms of neuropathic pain

*Correspondence: Dr Chih-Shung Wong, Department of Anesthesiology, Tri-Service General Hospital, no. 325, Chenggung Road, Section 2, Neihu, Taipei |14, Taiwan, Tel: +886 2 87927007, Fax: + 8862 87927009, E-mail: w82556@ndmctsgh.edu.tw

Received 21 September 2007; revised 18 December 2007; accepted 18 December 2007 remain controversial (Campbell and Meyer, 2006). Several possible mechanisms have been proposed, including (1) downregulation of $\mu$-opioid receptors, both pre- and postsynaptically after nerve injury (Kohno et al, 2005); (2) activation of voltage-gated calcium channel (Li et al, 2004); (3) increased trafficking of AMPA receptors to the cell surface that enhances glutamate release (Li et al, 2004); and (4) activation of neuroinflammation cascade (Raghavendra et al, 2002).

Pertussis toxin (PTX) is believed to ADP-ribosylate the $\alpha$-subunit of $\mathrm{Gi} / \mathrm{Go}$ proteins, thus disrupting the signal transduction of inhibitory Gi/Go-protein-coupled receptors. Intrathecal injection of PTX not only decreases the antinociceptive effect of opioid agonists but also produces thermal hyperalgesia and allodynia that appears similar to the symptoms reported in clinical patients who suffer from neuropathic pain (Womer et al, 1997; McCormack et al, 1998). This model had been used as an animal model for studying the pathophysiology of neuropathic pain (Womer et al, 1997). Similarly, studies had demonstrated that intrathecal PTX injection reduces the antinociceptive potency of etorphine and fentanyl (Shah et al, 1994), and selective $\mu$-agonist PL017 (Wong et al, 1992). Womer et al (1997) 
found that PTX disrupted the antinociceptive signal transduction of morphine, and therefore morphine is less effective in treating central and neuropathic pains than treating acute pain.

Opioid antagonists naloxone and naltrexone antagonize, at microgram doses, both excitatory and inhibitory opioid functions, while, at ultra-low nanogram doses, they enhance the analgesic effect of opioid agonists (Shen and Crain, 1997). Co-administration or post-treatment with an ultralow dose of naltrexone or naloxone was found to potentiate the antinociceptive effect of morphine on the neuropathic thermal hyperalgesia in rats (Powell et al, 2002), and extends the duration of analgesia in morphine-tolerant rats (Powell et al, 2002; Wang et al, 2005). The mechanism has been hypothesized to be due to the inhibition of the excitatory signaling of opioid receptors (Shen and Crain, 1997). Recent molecular evidence further confirms this hypothesis by demonstrating that ultra-low-dose naloxone, in combination with morphine, switches G-protein coupling, and signaling via $\mu$-opioid receptors, in chronic opiate-administrated animals (Wang et al, 2005). An opioid receptor can couple to both stimulatory and inhibitory $\mathrm{G}$ proteins; the $\mu$-opioid receptor-Gs coupling, following chronic morphine treatment was inhibited by ultra-lowdose naloxone (10 ng) co-treatment (Sudoh et al, 2003). Moreover, ultra-low-dose naloxone had also been used for the treatment of neuroinflammation (Liu and Hong, 2003) and inflammatory pain (Millan and Colpaert, 1990). These data suggest that ultra-low-dose naloxone might be clinically valuable in pain management. However, the exact effect of ultra-low-dose naloxone is still not clear in treating neuropathic pains. The present study was the first direct assessment of the effect of ultra-low-dose naloxone in antinociceptive and anti-neuroinflammatory effect in PTX-treated rats.

\section{MATERIALS AND METHODS}

\section{Animal Preparation and Intrathecal Drug Delivery}

Male Wistar rats (320-370 g) were anesthetized with phenobarbital (65 mg/kg, intraperitoneally) and implanted with an intrathecal catheter (day 0). In rats used for CSF dialysate collection, a microdialysis probe was also inserted via the atlanto-occipital membrane down to the lumbar enlargement L1-L2 of the spinal bony structure. The levels of L1-L2 spinal bony structure correspond to the spinal cord segments of L5, L6, and S1-S3, which are responsible for the tail-flick reflex (Grossman et al, 1982). After catheterization, all rats were returned to their home cages for recovery, each rat being housed individually and maintained on a $12 \mathrm{~h}: 12 \mathrm{~h}$ light-dark cycle with food and water freely available. Rats with neurological deficits were excluded. Three days after catheterization (day 3), PTX ( $1 \mu \mathrm{g}$ in $5 \mu \mathrm{l}$ saline; Calbiochem-Novabiochem International, San Diego, CA, USA) was injected via the intrathecal catheter to induce thermal hyperalgesia, and control rats were given saline instead. Four days later (day 7), saline or naloxone $(15 \mu \mathrm{g}$ or $15 \mathrm{ng}$ in $5 \mu \mathrm{l}$ saline; Sigma, Missouri, USA) was given intrathecally $30 \mathrm{~min}$ before saline or morphine $(10 \mu \mathrm{g}$ in $5 \mu \mathrm{l}$ saline; Sigma, Missouri, USA) injection. The use of rats in this study conformed to the
Guiding Principles in the Care and Use of Animals of the American Physiology Society and was approved by the National Defense Medical Canter Animal Care and Use Committee.

\section{Antinociception Test}

The antinociception test was modified and adapted from previous studies (Trujillo and Akil, 1991; Tai et al, 2006). Tail-flick latency was measured using the hot water immersion test $\left(52 \pm 0.5^{\circ} \mathrm{C}\right)$. The percentage of maximal possible antinociceptive effect (\% MPE) was calculated as ((maximum latency-baseline latency)/(cutoff latency-baseline latency) $) \times 100$. Latency of less than baseline or higher than $10 \mathrm{~s}$ was assigned MPE values of 0 or $100 \%$, respectively.

\section{Construction of Microdialysis Probe}

Spinal microdialysis probe construction was modified and adapted from previous study (Marsala et al, 1995). The probe was constructed using two $7 \mathrm{~cm}$ PE5 tubes $(0.008$ inch inner diameter, 0.014 inch outer diameter; Spectranetics, Colorado Springs, CO, USA) and a $4.2 \mathrm{~cm}$ cuprophan hollow fiber (Hospal, M100 per Set, France). To make the probe firm enough for implantation, a Nichrome-Formavar wire (0.0026 inch diameter; A-M System Inc., Everett, WA, USA) was passed through a polycarbonate tube $(102 \mu \mathrm{m}$ inner diameter, $194 \mu \mathrm{m}$ outer diameter; $0.7 \mathrm{~cm}$ in length) and a cuprophan hollow fiber, and then connected to a PE5 catheter with epoxy glue. The middle portion of the cuprophan hollow fiber was bent to form a U-shaped loop. The two ends of the dialysis probe, consisting of silastic tubes, were sealed with silicon sealant. The dead space of the dialysis probe was about $8 \mu \mathrm{l}$. During in vitro measurements, the recovery rate of the dialysis probe was $40 \%$ (refer to L-glutamate and L-aspartate) at room temperature with an infusion rate of $5 \mu \mathrm{l} / \mathrm{min}$. In our previous study, using this technique, it was possible to measure CSF amino acid level for up to 12 days after implantation (Wen et al, 2003b). Similarly, Marsala et al (1995) found that the recovery rate of dialysis probe for excitatory amino acids (EAAs) was $43 \%$ at day 12 .

\section{CSF Sample Collection and Measurement of EAAs}

On day 7, after treatment with drugs, rats were placed in restrainers without anesthesia, and the environment was kept dim and quiet for microdialysate collection. One of the externalized silastic tubes of microdialysis probe was connected to a syringe pump (CMA-100; Acton, Acton, MA, USA), and perfused with Ringer's solution $(0.33 \mathrm{mg} / \mathrm{ml}$ of calcium chloride, $8.6 \mathrm{mg} / \mathrm{ml}$ of sodium chloride, and $0.3 \mathrm{mg} / \mathrm{ml}$ of potassium chloride). Then CSF dialysates were collected from the other externalized silastic tube of the microdialysis probe. CSF dialysates were collected using a standard procedure of a 30-min washout period, followed by a 30 -min sample collection period at a flow rate of $5 \mu \mathrm{l} / \mathrm{min}$ in a polypropylene tube on ice, then frozen at $-80^{\circ} \mathrm{C}$ until assayed. 


\section{Amino Acid Analysis}

The dialysis samples were analyzed for amino acids by phenylisothiocyanate derivatization using an HPLC equipped (Agilent 1100; Agilent Technologies, Santa Clara, CA, USA) with a reverse-phase ZORBAX Eclipse AAA column $\left(4.6 \times 150 \mathrm{~mm}^{2}, 3.5 \mu \mathrm{m}\right)$ and fluorescent detector (Gilson model 121, set at $428 \mathrm{~nm}$ ) as described previously. External standards $(156.25,312.5,625,1250$, and $2500 \mu \mathrm{M}$ of authentic amino acids) were run at the beginning and the end of each sample group. Peak heights were normalized to standard peaks and then quantified based on a linear relationship between peak height and amounts of corresponding standards.

\section{Spinal Cord Sample Preparation and Western Blotting Analysis}

The dorsal portion of the lumbar spinal cords was homogenized in RIPA lysis buffer (Upstate Inc., Lake Placid, NY, USA), the homogenates were centrifuged at $10000 \mathrm{~g}$ for $30 \mathrm{~min}$ at $4^{\circ} \mathrm{C}$, and the supernatants were used for western blotting. Samples were denatured by heating at $95^{\circ} \mathrm{C}$ for $10 \mathrm{~min}$, then separated on a $10 \%$ SDS-PAGE gel, and transferred to a nitrocellulose membrane (Bio-Rad). The membrane was blocked with 5\% non-fat milk and incubated overnight at $4^{\circ} \mathrm{C}$ with polyclonal guinea-pig anti-rat GLT-1 or anti-rat GLAST antibodies, polyclonal rabbit anti-rat EAAC1 antibodies (all from Chemicon, Temecula, CA) or anti-rat glutamate dehydrogenase (GDH) antibodies (US Biological), monoclonal mouse anti-rat glutamine synthetase (GS) antibodies (Chemicon), then with the corresponding horseradish peroxidaseconjugated donkey anti-guinea-pig or anti-rabbit IgG or goat anti-mouse IgG secondary antibody (all from Chemicon) for $1 \mathrm{~h}$ at room temperature. After reaction with ECL solution (Amersham, Arlington Heights, IL, USA), a bound antibody was visualized using a chemiluminescence imaging system (Syngene, Cambridge, UK). Finally, the blots were incubated at $56^{\circ} \mathrm{C}$ for $18 \mathrm{~min}$ in stripping buffer $(0.0626 \mathrm{M}$ Tris- $\mathrm{HCl}, \mathrm{pH}$ 6.7, 2\% SDS, $0.1 \mathrm{M}$ mercaptoethanol) and re-probed with a monoclonal mouse anti- $\beta$-actin antibody (Sigma) as the loading control. The optical density of each specific band was measured using a computerassisted imaging analysis system (Gene Tools Match software; Syngene).

\section{Immunocytochemistry and Image Analysis}

Rats were killed by exsanguinations under isoflurane anesthesia, the lumber enlargement (L5-S3) of the spinal cord was immediately removed and embedded in optimal cutting temperature compound (Sakura Finetec Inc., Torrance, CA, USA). The sections $(5 \mu \mathrm{m})$ were fixed by immersion in ice-cold acetone/methanol $(1: 1)$ for $5 \mathrm{~min}$. After washing in ice-cold phosphate-buffered saline, the sections were incubated sequentially with FITC-labeled mouse monoclonal anti-rat OX42 antibody (for microglia; Serotec, Oxford, UK; green fluorescence), and unlabeled goat polyclonal antibodies against one of rat TNF- $\alpha$, rat IL$1 \beta$, and rat IL-6 (all from R\&D System, Minneapolis, MN, USA) at $4^{\circ} \mathrm{C}$ overnight. The sections were then reacted with rhodamine-labeled donkey anti-goat $\operatorname{IgG}$ antibody at room temperature for $1 \mathrm{~h}$ (Santa Cruz Biotechnology Inc., Santa Cruz, CA, USA). An Olympus BX 50 fluorescence microscope was used for image capture (Olympus Optical, Tokyo, Japan), and a Delta Vision disconsolation microscopic system operated by SPOT software (Diagnostic Instruments Inc., Sterling Heights, MI, USA) was used.

\section{Quantitative Real-Time PCR}

Total RNA extraction and cDNA synthesis. Total RNA was extracted using the TRIzol reagent (Invitrogen, Carlsbad, $\mathrm{CA}$ ), according to the manufacturer's instructions. Samples were treated with DNase (Invitrogen) to remove contaminating DNA, the concentration and purity was determined by measuring the absorbance at 260 and $280 \mathrm{~nm}$ with spectrophotometer.

Total RNA was reverse transcribed into cDNA using the Superscript II First Strand Synthesis system from Invitrogen. cDNA was synthesized using $1 \mu \mathrm{g}$ of total RNA, $50 \mathrm{ng}$ of random hexamer primer, $0.5 \mathrm{mM}$ dNTP mix, $10 \mathrm{mM}$ dithiothreitol, $1 \times$ RT buffer and $200 \mathrm{U}$ of Superscript II reverse transcriptase in a total volume of $20 \mu \mathrm{l}$. The reaction was carried out at $42^{\circ} \mathrm{C}$ for $50 \mathrm{~min}$ and terminated by deactivation of the enzyme at $70^{\circ} \mathrm{C}$ for $15 \mathrm{~min}$. Control reactions, lacking either reverse transcriptase or template, were included to assess carryover of genomic DNA and RNA contamination, respectively.

Quantitative real-time PCR. Amplification of cDNA was performed using the Applied Biosystems Prism 7000 Sequence Detection system and TaqMan ${ }^{\circledR}$ Universal PCR master mix (SYBR Green I), according to the manufacturer's specifications (Applied Biosystems Inc., Foster City, CA). The gene-specific probes were labeled at the $5^{\prime}$ end with the reporter dye FAM, and the GAPDH internal control probe was labeled at the $5^{\prime}$ end with the reporter dye VIC. A non-fluorescent quencher and the minor groove binder were added to the $3^{\prime}$ end of each probe as quenchers. The thermal cycle conditions were $10 \mathrm{~min}$ at $95^{\circ} \mathrm{C}$, and two-step PCR for 40 cycles of $95^{\circ} \mathrm{C}$ for $15 \mathrm{~s}$, followed by incubation at $60^{\circ} \mathrm{C}$ for $1 \mathrm{~min}$. All samples were tested in triplicate. The amplification data were analyzed by using Applied Biosystems Prism Sequence Detection Software version 1.1 (Applied Biosystems). To compare the relative expression levels with different treatments, the expression of the gene of interest was normalized to that of the GAPDH control using the $\Delta \Delta C_{\mathrm{T}}$ method recommended by the manufacturer. The primers were designed using primer express software and shown in Table 1 .

\section{Statistical Analysis of Data}

Data from the tail-flick withdrawal latency are presented as mean \pm SEM, and analyzed by one-way ANOVA, followed by multiple comparisons with the Student-Newman-Keuls post hoc test. A significant difference was defined as a $P$-value $<0.05$. 
Table I Sequence of PCR Primers Specific for the GLAST, GLT-I, EAACI, GS, and GDH Genes

\begin{tabular}{lll}
\hline Target & Forward & Reverse \\
\hline GLAST & CGGGATTGTGAACACTTGTC & ACGGGTTTCTCTGGTTCATTG \\
GLT-I & TTGTCTATCACCTITCCAAGTC & AGTTGCTTCCCTGTGGTT \\
EAACI & CAGGTGGATGTCTCCTCTGAAGT & GAACGAGATGGTGTCAGATTTGTCTA \\
GS & GTTTGGAATGGACAGGAGTATA & TCCCACACCGCAGTAATAG \\
GDH & AAATATGAAAGGGACTCGAACTA & TCAGATGCACCCGATATTC \\
TNF- $\alpha$ & CACCGGCAAGGATTCCAA & CACTCAGGCATCGACATTCG \\
IL-I $\beta$ & AGCCTTTGTCCTCTGCCAAGT & CCAGAATGTGCCACGGTTT \\
IL-6 & TGTTCTCAGGGAGATCTTGGAAT & CATCGCTGTTCATACAATCAGAATT \\
\hline
\end{tabular}

\section{RESULTS}

\section{Number of Rats Used in the Experiments}

In the present study, all rats $(n=184)$ were intrathecally implanted with either an intrathecal catheter only $(n=139)$ or in combination with a microdialysis probe $(n=45)$ for the following experiments. The rats with an intrathecal catheter only, for the administration of drugs, were used for behavioral nociceptive tail-flick test; then rats were killed for western blot $(n=78)$, for IHC $(n=30)$, and for RT-PCR $(n=31)$. For CSF dialysate collection and EAA measurement, rats $(n=45)$ were implanted with another microdialysis probe, in addition to the intrathecal catheter. Rats with either neurological deficit or obstructed microdialysis probe were excluded from the experiments.

\section{Ultra-Low-Dose Naloxone Restores the Antinociceptive Effect of Morphine in PTX-Treated Rats}

In naïve rats, the average baseline tail-flick latency of the hot water immersion test was $1.9 \pm 0.25 \mathrm{~s}(n=20)$. In animals tested 4 days after intrathecal PTX injection, thermal hyperalgesia and reduction in the antinociceptive effect of morphine were observed (Figure $1 \mathrm{a}$ and $\mathrm{b}$ ). Single intrathecal injection of ultra-low-dose naloxone $(15 \mathrm{ng}$ in $5 \mu \mathrm{l}$ saline) produced a slight, but not significant, reduction in the thermal hyperalgesia in PTX-treated rats (Figure 1a). As shown in Figure 1b, the maximum antinociceptive effect of morphine ( $10 \mu \mathrm{g}$ in $5 \mu \mathrm{l}$ saline, intrathecal injection) was seen at $60 \mathrm{~min}$ after morphine injection in naïve rats and this effect was blocked by the PTX treatment; pretreatment of the classical dose of naloxone $(15 \mu \mathrm{g}$ in $5 \mu \mathrm{l}$ saline, intrathecal injection) had no effect on the antinociceptive effect of morphine. In contrast, pretreatment of PTX-treated rats with ultra-low-dose naloxone $30 \mathrm{~min}$ before morphine injection restored the antinociceptive effect of morphine ( $\sim 75-95 \%$ of the morphine effect in naïve rats).
Pretreatment with Ultra-Low-Dose Naloxone Followed by Morphine Administration Suppresses EAA Release in PTX-Treated Rats

As in our previous study (Sung et al, 2005), PTX injection induced a significant increase in the concentration of EAAs L-aspartate and L-glutamate in the CSF dialysates (Figure 2a and $b$ ). Intrathecal injection of either morphine or naloxone alone did not inhibit the PTX-induced EAA release, and no additional effect was observed by the pretreatment with micrograms of naloxone. In contrast, pretreatment of the ultra-low-dose naloxone $30 \mathrm{~min}$ before morphine injection completely blocked the PTX-induced increase in L-aspartate and L-glutamate levels and further inhibited the EAA concentrations lower than the baseline level (Figure 2a and $b$ ). The peak effect was observed at $60 \mathrm{~min}$ after morphine injection, which corresponded to the maximal effect of ultra-low-dose naloxone on the antinociceptive effect of morphine in PTX-treated rats (Figure 1b).

Effect of Ultra-Low-Dose Naloxone on the Protein and mRNA Expression of L-Glutamate Transporters and Glutamate-Metabolizing Enzyme in PTX-Treated Rats

PTX-treated rat spinal cords showed downregulation of all three L-glutamate transporters (GTs) (GLAST, GLT-1, and EAAC1), GS, and GDH (Figure 3a and b). Intrathecal administration of morphine or naloxone alone, or a regular dose of naloxone plus morphine injection did not produce any effect on either the protein or mRNA expression of the PTX-treated spinal cords, whereas pretreatment with ultralow-dose naloxone, $30 \mathrm{~min}$ prior to morphine injection, restored the protein expression of all three GTs and GS, but not that of GDH (Figure 3a and b). Moreover, in the timecourse study (Figure 4a and b), the effect of ultra-low-dose naloxone pretreatment plus morphine administration on the restoration of all three GTs and GS protein expression, but not that of GDH, peaked at $60 \mathrm{~min}$ after morphine 

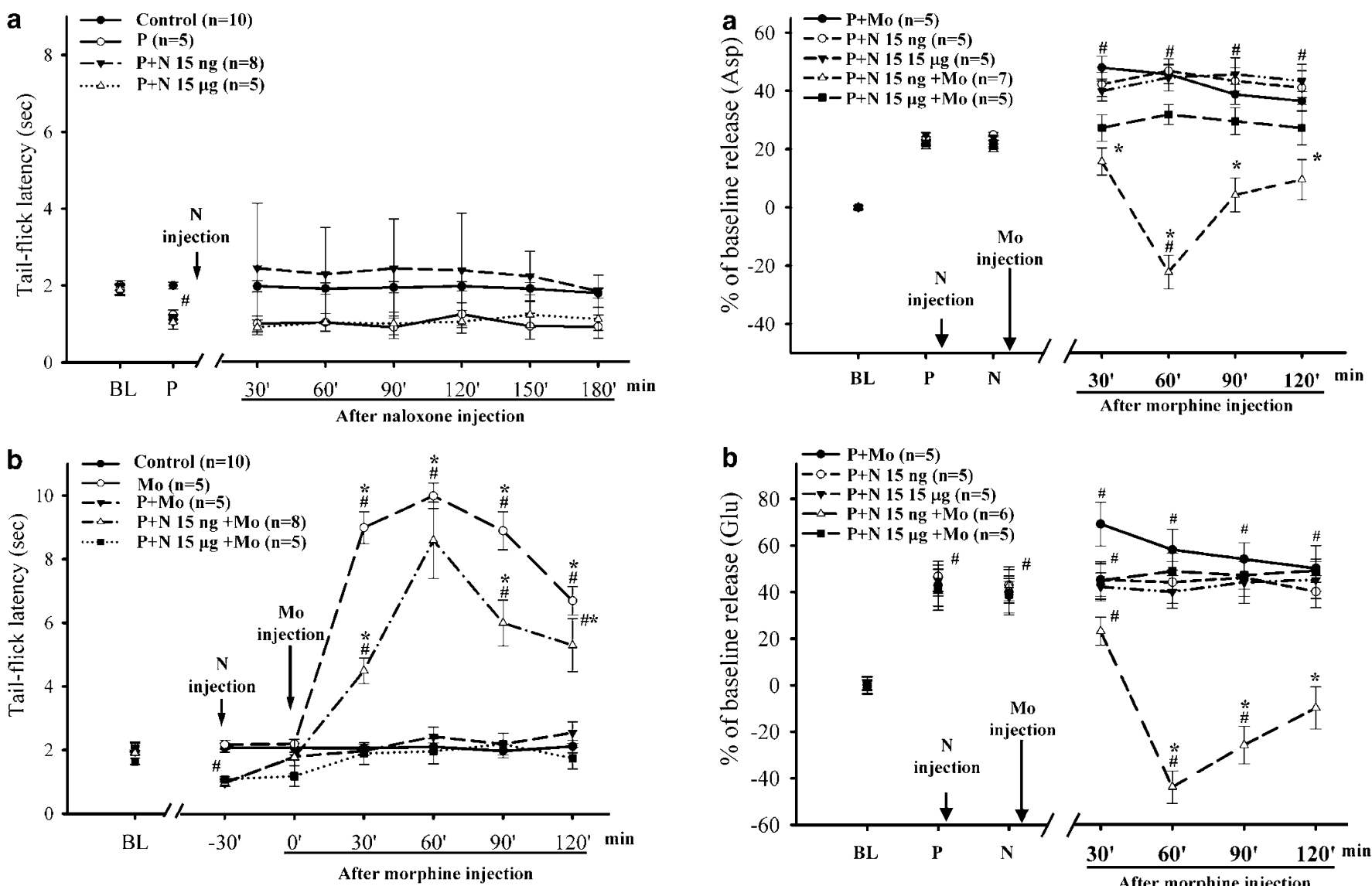

Figure I Ultra-low-dose naloxone restores the antinociceptive effect of morphine in PTX-treated rats. On day 7, after treatment with drugs, rats were placed in plastic restrainers and environment was kept dim and quiet for antinociception testing. Rats with the baseline latency $>6 \mathrm{~s}$ were excluded from these tests and a 10 -s cutoff was used to minimize tissue damage. (a) Following either pertussis toxin ( I $\mu$ g in $5 \mu \mid$ saline) or saline (control) injection, the antinociceptive effect was examined and expressed by tail-flick latency; naloxone alone (either I $5 \mu$ g or I $5 \mathrm{ng}$ in $5 \mu$ | saline intrathecal injection) provided no antinociceptive effect. ${ }^{*} P<0.05$ when compared to the control group. (b) The effect of naloxone pretreatment on the antinociceptive effect of morphine $(10 \mu \mathrm{g}$ in $5 \mu \mathrm{l}$ saline, intrathecal injection) was examined on day 4 after intrathecal injection of either pertussis toxin or saline. ${ }^{\#} P<0.05$ when compared to the control group; ${ }^{*} P<0.05$ when compared to the pertussis toxin group. Data are expressed as mean \pm SEM and averaged from the indicated number of rats. BL, baseline value; $\mathrm{P}$, pertussis toxin, value at 4 days after PTX injection; N, naloxone.

injection and lasted for at least $120 \mathrm{~min}$. However, the mRNA expression of the GTs, GS, and GDH had no significant change in PTX-treated rats, and ultra-low-dose naloxone plus morphine administration also had no effect on the mRNA expression (Figure 5).

\section{Ultra-Low-Dose Naloxone Inhibits Microglial Activation, Pro-Inflammatory Cytokine Production and mRNA Expression in PTX-Treated Rats}

In control rat spinal cords, a weak immunostaining (OX42positive cells, shown in green) was observed in resting microglia with a small cell body and long processes (Figure $6 \mathrm{a}, \mathrm{d}$, and g). In contrast, an intense immunoreactivity was

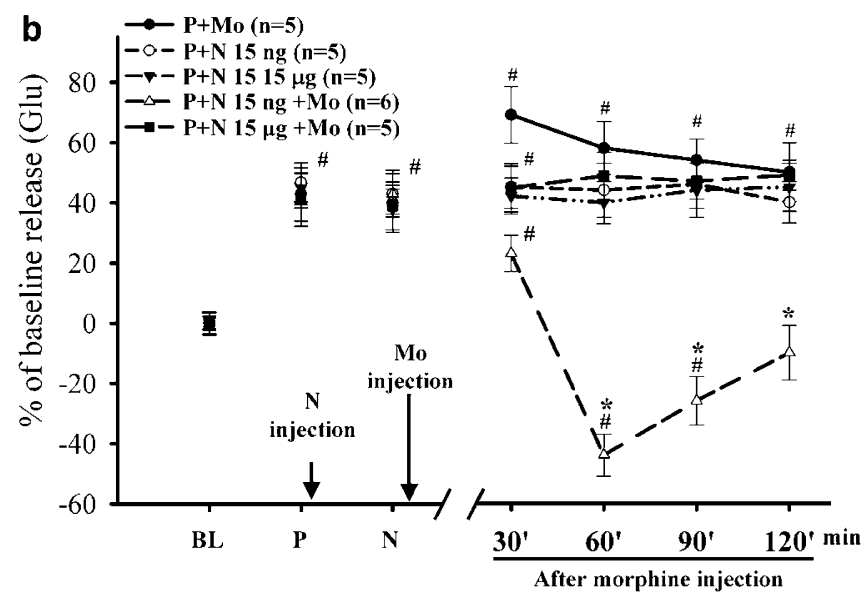

Figure 2 Ultra-low-dose naloxone treatment before morphine injection suppresses EAA release in PTX-treated rats. Basal CSF dialysates were collected on day I before intrathecal PTX injection, and the 'P' sample was collected on day 4 after PTX injection. Then the rats received either naloxone or saline injection and ' $\mathrm{N}$ ' sample was collected $30 \mathrm{~min}$ later. After $30 \mathrm{~min}$, following morphine or saline injection, the time-course samples were collected. A further four 30-min samples were collected for 120 min after morphine injection. The concentrations of L-aspartate (a) and L-glutamate (b) in the dialysates were measured by HPLC as described in the Materials and Methods. Data are presented as mean \pm SEM and averaged from the indicated number of rats. ${ }^{\#} P<0.05$ when compared to the baseline levels; $* P<0.05$ when compared to the ' $P$ ' levels.

observed in activated microglia with a large cell body and retracted processes in PTX-treated rat spinal cords (Figure $6 \mathrm{~b}$, e, and h). On day 4 after PTX treatment, the sections stained for cytokines (TNF- $\alpha$, IL-1 $\beta$, and IL-6) and microglia were overlaid, except for TNF- $\alpha$ (Figure 6e), colocalization of IL-1 $\beta$ and IL-6 with OX42 immunoreactivity (white arrows) was observed in the spinal cord sections from PTX-treated rats (Figure $6 \mathrm{~b}$ and $\mathrm{h}$ ), which indicated that these cytokines were expressed in activated microglia, but not neurons. The PTX-induced microglial activation and increase in IL- $1 \beta$ and IL- 6 expression were inhibited by ultra-low-dose naloxone plus morphine administration, which supports the idea that this expression in the spinal cord of PTX-treated rats might play an important role in the PTX-induced thermal hyperalgesia. As shown in Figure 7 , the mRNA expression of the proinflammatory cytokines, TNF- $\alpha$, IL- $1 \beta$, and IL-6, was significantly increased in the PTX-treated rats, and this 


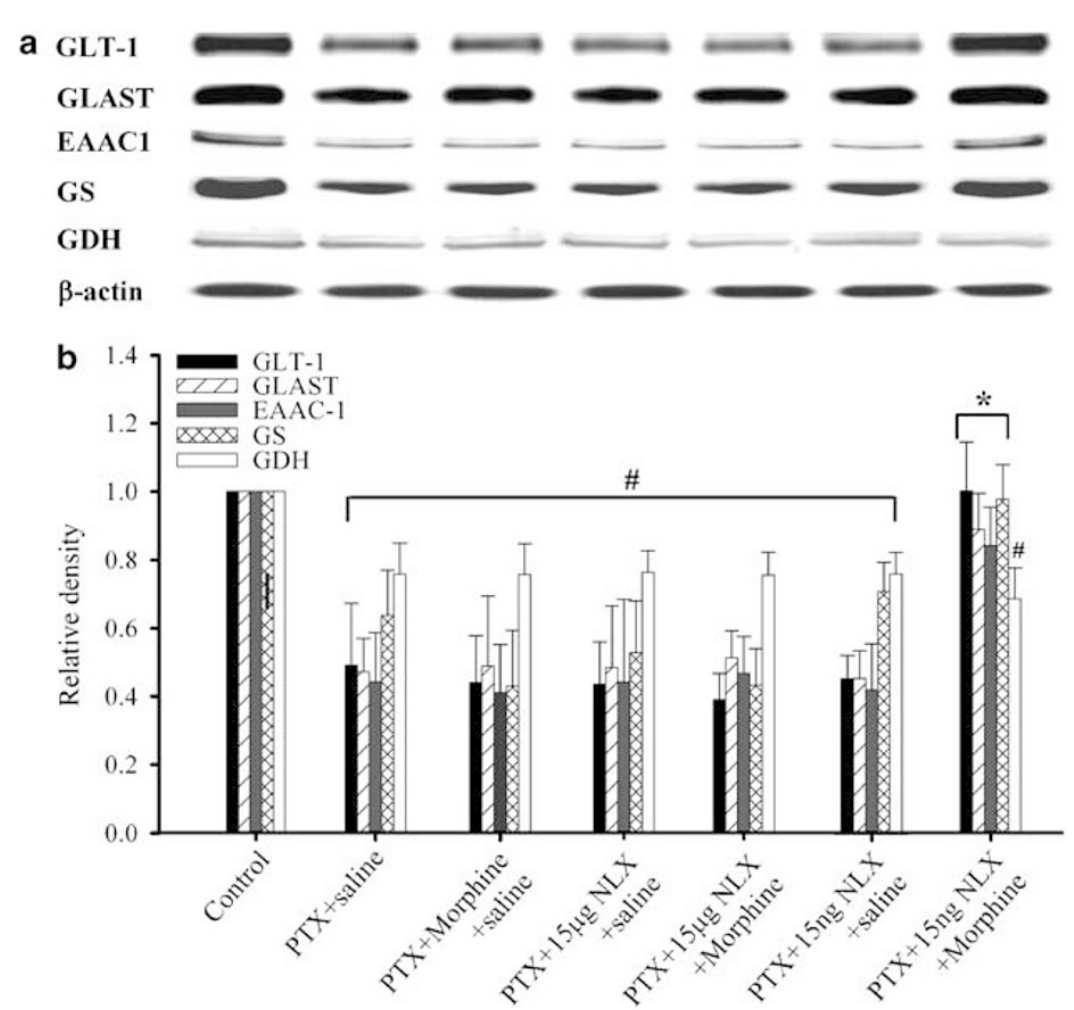

Figure 3 Ultra-low-dose naloxone treatment before morphine administration upregulates protein expression of GTs and GS, but not GDH, in PTX-treated rat spinal cords. On day 7, after drug administration as described above, rats were killed by exsanguinations under isoflurane anesthesia. Laminectomy was performed at the lower edge of the 12th thoracic vertebra, and L5-S3 segment of the spinal cord was removed and separated into ventral and dorsal parts immediately for western blot analysis. (a) Western blot analysis of GTs, GS, and GDH in the spinal cord dorsal horn of the indicated treatment groups of rats at $120 \mathrm{~min}$ after the final morphine injection. (b) Quantification of the data from (a). $\beta$-Actin was used as the loading control. Data are averaged from six rats per group with $15 \mu \mathrm{g}$ total sample protein for each lane. ${ }^{\#} P<0.05$ compared to the control group; ${ }^{*} P<0.05$ compared to the PTX group. NLX, naloxone.

effect was significantly attenuated by the ultra-low-dose naloxone treatment before morphine administration.

\section{DISCUSSION}

In the present study, ultra-low-dose naloxone pretreatment not only restored the antinociceptive effect of morphine but also suppressed the PTX-evoked increase in L-aspartate and L-glutamate levels in the spinal CSF dialysates. Downregulation of spinal GTs, GS, and GDH protein expression, with the exception of GDH, was also reversed by ultra-lowdose naloxone treatment before morphine administration in PTX-treated rats. This reversal was correlated with the restoration of the antinociceptive effect of morphine in PTX-treated rats. Moreover, activation of microglia and increase in pro-inflammatory cytokine expression (TNF- $\alpha$, IL-1 $\beta$, and IL-6) were observed in PTX-treated rat spinal cords, and both were inhibited by ultra-low-dose naloxone plus morphine administration. Interestingly, each single drug treatment, including ultra-low-dose naloxone had no effect on the alterations by the PTX treatment in rats.

Recently, we were able to demonstrate a correlation between morphine's analgesic effect and CSF EAA concentrations in terminal cancer patients having pain (Wong et al, 2002). At the analgesic dose of morphine, a lower concentration of EAAs was observed in the CSF; an increase in EAA levels in the CSF was observed when morphine lost its analgesic effect after long-term intrathecal administration for pain control in terminal cancer patients. Furthermore, doubling the effective dose of morphine neither provided satisfactory pain relief nor reduction in CSF EAA concentrations (Wong et al, 2002). This result suggests that the loss of morphine's analgesic effect is directly related to an increase in EAAs in the CSF. Moreover, our previous studies found that the development of morphine tolerance and neuropathic pain might be linked to an increase in EAA release and subsequent activation of NMDA receptors (Liu et al, 2000; Wen et al, 2003a). Similarly, in the present study, intrathecal PTX injection induced thermal hyperalgesia in rats and accompanied by a prolonged increase in the EAA L-glutamate and L-aspartate concentrations in the spinal CSF dialysates and loss of the antinociceptive effect of morphine. This increase in the EAA levels after PTX injection, observed in our present study, might be due to downregulation of GTs, which limited the re-uptake of the EAAs released. Ultra-low-dose naloxone pretreatment inhibited PTX injection-induced EAA elevation, thus restoring the antinociceptive effect of morphine. These results provide direct evidence of the correlation of EAA levels and the antinociceptive effect of morphine.

L-Glutamate, the principal excitatory neurotransmitter in neuronal circuitry, is critically involved in pain modulation, opioid tolerance, dependence, and withdrawal (Wong et al, 

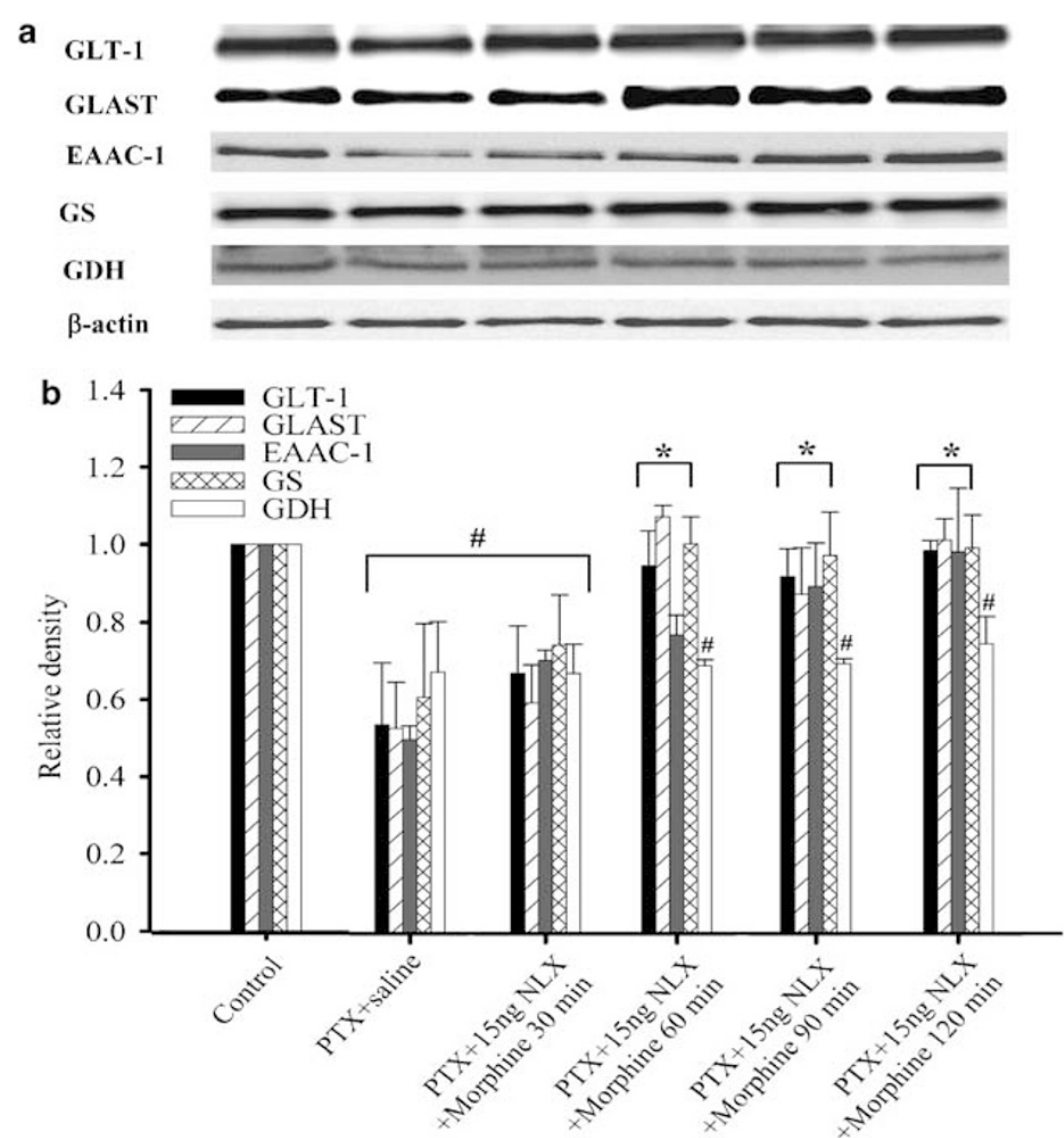

Figure 4 Time course of ultra-low-dose naloxone pretreatment plus morphine administration on the GTs and GS protein expression in PTX-treated rat spinal cord dorsal horns. (a) Time course of GTs, GS, and GDH protein expression in the spinal cord after ultra-low-dose naloxone pretreatment plus morphine administration in PTX-treated rats. Spinal cord samples were collected at 30,60, 90, and I 20 min after morphine injection. (b) Quantification of the data from (a). $\beta$-Actin was used as the loading control. Data are averaged from six rats per group with $15 \mu \mathrm{g}$ total sample protein for each lane. ${ }^{\sharp} P<0.05$ when compared to the control group; $* P<0.05$ when compared to the PTX group.

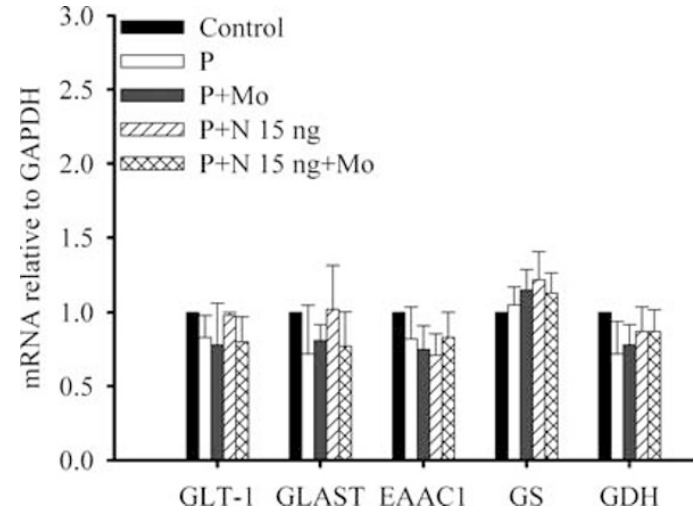

Figure 5 The mRNA expression of L-glutamate transporters, GS, and GDH in PTX-treated rat spinal cord dorsal horns after 120 min of final morphine injection. On day 7, after drug administration as described above, rats were killed by exsanguinations under isoflurane anesthesia. Laminectomy was performed at the lower edge of the 12th thoracic vertebra, and L5-S3 segment was removed for quantitative real-time PCR examination. There were no significant differences in the mRNA expression of L-glutamate transporters, GS, and GDH at each examined groups ( $n=3$ of each group).

1998; Tai et al, 2006). There are two major mechanisms for cell protection against L-glutamate-induced neurotoxicity: (a) removal of synaptic L-glutamate by high-affinity uptake of GTs and (b) metabolism and recycling of L-glutamate by synaptic astrocytes via GS (Danbolt, 2001). To ensure a high signal-to-noise ratio for synaptic signaling and to protect neurons, the extracellular concentration of EAAs in the synaptic junction needs to be maintained at low levels $(<1 \mu \mathrm{M})$, and GTs, particularly the glial GTs, play an important role in maintaining the synaptic EAA concentrations at physiologic levels (Danbolt, 2001). Recent evidence shows that downregulation of the protein expression of GTs contributes to morphine tolerance (Sung et al, 2005; Tai et al, 2006), dependence (Ozawa et al, 2001), and inflammatory pain (Sung et al, 2004). Moreover, a decrease in GLT-1 mRNA level is observed in the brain of naloxoneprecipitated morphine withdrawal in rats (Ozawa et al, 2001). The present study found that PTX treatment downregulated protein expression of GTs, but not mRNA expression. It supports the idea that PTX might only affect either the translation or post-translational level of GTs, but not the transcription level. In our present study, similar to the previous study (Mao et al, 2002), both the neuronal (EAAC1) and glial (GLAST and GLT-1) GTs were downregulated in the PTX-treated rat spinal cords. Furthermore, we found that ultra-low-dose naloxone treatment before morphine administration reversed the downregulation of GLAST, GLT-1, and EAAC1 and even slightly increased GLT-1 expression, thus enhancing the re-uptake of synaptic EAAs and restoring the antinociceptive effect of morphine 
Control
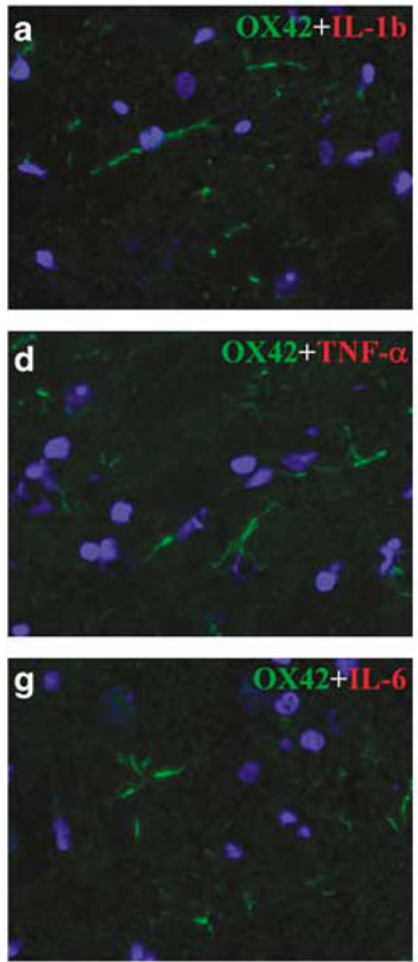

$P$
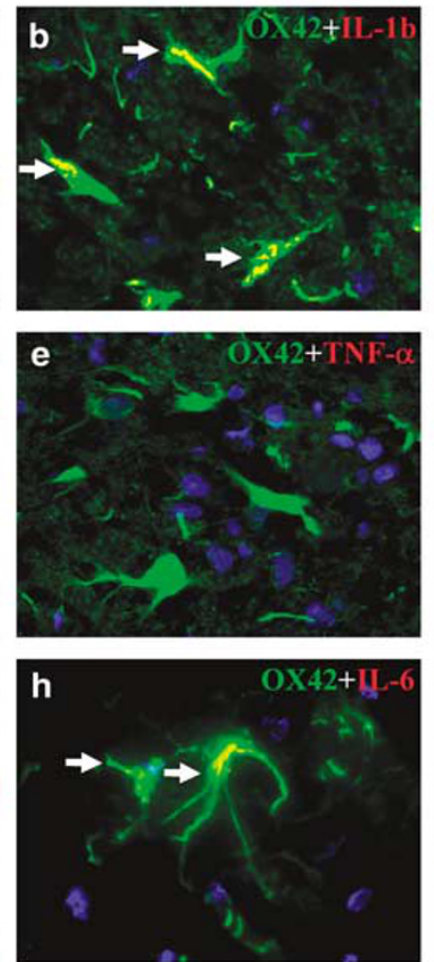

$\mathrm{P}+\mathrm{N}+\mathrm{Mo}$
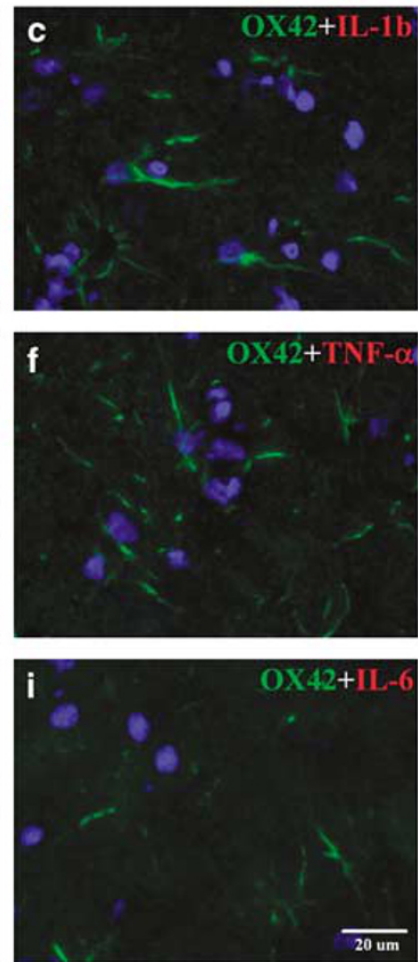

Figure 6 Ultra-low-dose naloxone plus morphine inhibit microglial activation. On day 7, after drug administration as described above, rats were killed by exsanguinations under isoflurane anesthesia. Laminectomy was performed at the lower edge of the 12th thoracic vertebra, and L5-S3 segment was removed and cut for immunocytochemistry analysis. OX42 staining for microglia is shown in green for IL-I $\beta$ (top row), TNF- $\alpha$ (middle row), and IL-6 (bottom row) in red; overlap of the two labels is demonstrated in yellow (white arrows). Left panels, saline controls; center panels, PTX-treated rats; right panels, ultra-low-dose naloxone plus morphine intrathecal injection in PTX-treated rats. Overlap of IL-I $\beta$ and IL-6 with OX42 was observed in PTXactivated microglia, but not in the microglia 120 min later by the ultra-low-dose naloxone plus morphine treatment of rat spinal cord dorsal horns. Scale bar represents $20 \mu \mathrm{m}$.

in PTX-treated rats. These results provide direct evidence of the relationship between expression of GTs and the antinociceptive effect of morphine.

Several lines of evidence suggest that GLAST and GLT-1 are the predominant GTs and are responsible for clearing synaptic glutamate and preventing excitotoxicity (Tanaka et al, 1996; Robinson and Dowd, 1997). Previous study has shown that many regulatory factors, such as dibutyryl cAMP, epidermal growth factor, and pituitary adenylate cyclase-activating polypeptide, modulate GLAST and GLT-1 mRNA levels (Zelenaia and Robinson, 2000). Gene deletion, gene knockdown, and pharmacological studies had demonstrated that GLT-1 played more important role than GLAST in the clearance of extracellular glutamate (Robinson and Dowd, 1997). Knockdown of spinal GLT-1 decreased the threshold of nociceptive stimuli by formalin injection in rat hindpaw (Niederberger et al, 2003). Ozawa et al (2001) observed a significant reduction in the GLT-1 mRNA expression in morphine-dependent rats. Sitcheran et al (2005) found that NF- $\kappa \mathrm{B}$ directly interacts and regulates GLAST and GLT-1 promoters. In the present study, treatment with ultra-low-dose naloxone before morphine administration significantly upregulated the protein expression of GTs, but not mRNA expression in PTX-treated rats. This result suggests that ultra-low-dose naloxone plus morphine administration might be regulated by the protein synthesis of GTs. As is already known, L-glutamate removed from the synaptic cleft by astrocytes undergoes a number of potential fates, including conversion to glutamine by GS and L-glutamate metabolism via ATP-generating oxidative deamination GDH-TCA cycle pathway when extracellular L-glutamate level is high. GS is located mainly in the astrocytes of CNS; the primary role of astrocytes is to protect neurons from glutamate excitotoxicity by taking up excess L-glutamate through GLAST and GLT-1 and converting glutamate into glutamine by GS (Suarez et al, 2002). GDH is demonstrated in synaptic terminals and neuronal mitochondria; it is mainly responsible for converting L-glutamate into $\alpha$-ketoglutarate in the CNS (Danbolt, 2001). GS and GDH are significantly reduced in multiple sclerosis (Werner et al, 2001) and allergic encephalomyelitis (Hardin-Pouzet et al, 1997). In the present study, we found that GS and GDH protein levels were reduced in PTX-treated rats, and ultra-low-dose naloxone treatment before morphine administration reversed the expression of GS, but not GDH. This suggests that GS located in the astrocytes regulates the glutamateglutamine cycle in the spinal cord, causes a reduction in L-glutamate concentration in the astrocytes, which in turn enhances the L-glutamate re-uptake by GTs and reduces the synaptic L-glutamate concentration, and subsequently reduces L-glutamate-mediated pathologic effects.

Previous studies have shown that neuropathic pain enhances pro-inflammatory cytokine (TNF- $\alpha$, IL- $1 \beta$, and 


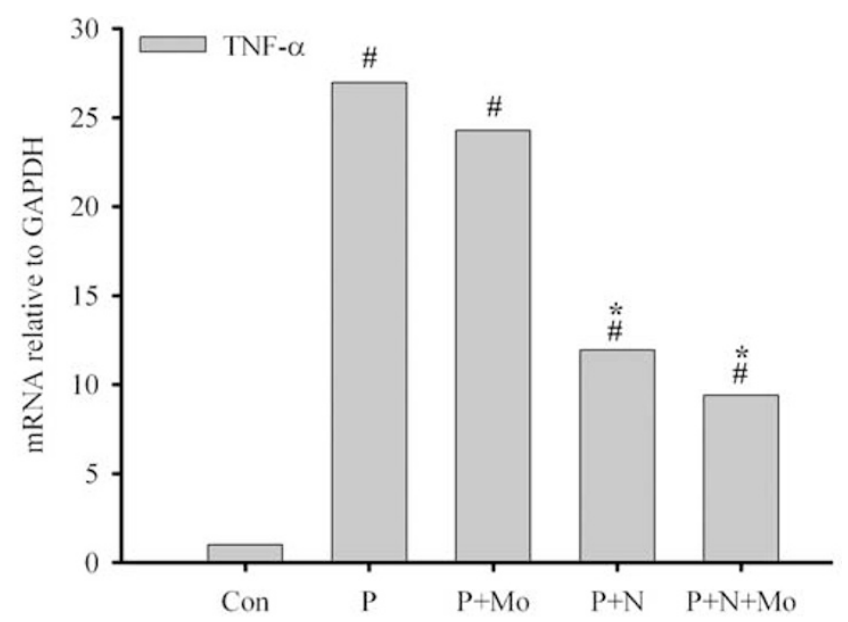

was also observed (Okada et al, 2005). This mechanism may be via the inhibition of tyrosine kinase receptors, or intracellular mitogen-activated protein kinase (Danbolt, 2001), or IKK, which triggers $\mathrm{I} \kappa \mathrm{B}$ phosphorylation and degradation, thereby causing NF- $\kappa \mathrm{B}$ translocation and DNA binding, subsequently repressing GLT-1 transcription and expression (Sitcheran et al, 2005). In our present study, ultra-low-dose naloxone effectively attenuated the PTXinduced elevation of cytokine protein (IL-1 $\beta$ and IL-6) and mRNA (TNF- $\alpha$, IL-1 $\beta$, and IL-6) expression. Therefore, we suggest that ultra-low-dose naloxone might act mainly via inhibiting the protein and mRNA expression of proinflammatory cytokines. It not only reduces the accumulation of synaptic EAAs but also further reduces EAA concentrations lower than the basal level, which might be responsible for restoring the analgesic effect of morphine by ultra-low-dose naloxone pretreatment in PTX-treated rats. In the spinal cord, astrocytes and microglia are activated in response to diverse noxious stimuli and release of proinflammatory cytokines, such as TNF- $\alpha$, IL- $1 \beta$, and IL-6, and affect cells far from the release site in a paracrine manner, and enhance the release of pain-related transmitters or substances (eg NO, prostaglandins, and EAA) from the primary afferent terminals (Watkins et al, 2003). Administration of glial metabolic inhibitors, cytokine antagonists, and anti-inflammatory cytokine IL-10 has been shown to attenuate the development of opioid tolerance and prevent hyperalgesia and allodynia (Raghavendra et al, 2002). In L5 spinal nerve-transfected rats, chronic subcutaneous morphine administration increases microglial and astrocytic immunoreactivity, and pro-inflammatory cytokine mRNA and protein expression; similarly, neuroimmune activity was greater in rats with nerve injury and morphine co-administration than rats with nerve injury only (Raghavendra et al, 2002). This indicates a possible synergistic effect of opioid administration and neuropathy on glial activation and cytokine production. A number of animal studies had found that ultra-low-dose naloxone inhibited the release of pro-inflammatory cytokines and superoxide generation from LPS-activated microglia and the resident macrophages within the nervous system (Liu et al, 2000; Liu and Hong, 2003). Similarly, the present study shows that intrathecal administration of ultra-low-dose naloxone plus morphine effectively attenuated PTX-induced glial activation and cytokine expression, which may be responsible for the enhancement of antinociceptive effect of morphine in PTX-treated rats.

In our previous study, we had demonstrated that PTX completely disrupted inhibitory G-protein coupling to opioid receptors, which led to blockade of the classical inhibitory opioid receptor signaling (Wong et al, 1992); this might be the reason for the lack of antinociceptive effect by the intrathecal morphine injection, and as known, the opioid receptor antagonist naloxone, at the classical high dose, had no antinociceptive effect in neither naïve nor PTX-treated rats. In contrast, Smart and Lambert (1996) had demonstrated that opioids, at doses well below those producing neuronal inhibition, exerted stimulatory effects. Moreover, in cultured dorsal root ganglion neurons, nanomolar concentration of opioid agonist diprenorphine $(10 \mathrm{nM})$ increased the action potential duration, whereas micromolar concentration produced the opposite effect 
(Chen et al, 1988; Shen and Crain, 1997). In this model, they concluded that ultra-low-dose morphine $(0.1 \mu \mathrm{g} / \mathrm{kg}$, s.c.) activates a Gs-coupled mode of the opioid receptor action, which activates adenylyl cyclase and increases neuronal excitability that produced hyperalgesia (Shen and Crain, 1997) and were blocked by ultra-low doses (10 ng/kg, s.c.) of opioid receptor antagonist naltrexone (Crain and Shen, 1995). Furthermore, Shen and Crain (1997) found that systemic administration of ultra-low-dose naltrexone selectively antagonized the stimulatory action of morphine, which potentiated morphine-induced analgesia and inhibited the development of tolerance and physical dependence. Similarly, in our present study, hyperalgesia, with a tail-flick latency of $0.89 \pm 0.2 \mathrm{~s}$, was induced by the intrathecal PTX injection, and ultra-low-dose naloxone ( $15 \mathrm{ng}$ in $5 \mu \mathrm{l}$ saline) shifted this latency to $2.6 \pm 1.1 \mathrm{~s}$ near to the baseline $(1.9 \pm 0.25 \mathrm{~s})$ of naïve rats; in contrast, a high dose of naloxone ( $15 \mu \mathrm{g}$ in $5 \mu \mathrm{l}$ saline) had no effect on the baseline latency of PTX-treated rats. Taken together, the effect was suggested to be due to the inhibition of opioid excitatory signaling (Shen and Crain, 1997). Recently, Wang et al (2005) found that ultra-low-dose naloxone plus morphine treatment inhibit the switching of G-protein-coupled $\mu$-opioid receptor signaling from inhibition to excitation in chronic opiate-administrated rats (Wang et al, 2005). Furthermore, in our preliminary immunoprecipitation data, ultra-low-dose naloxone alone slightly restored the coupling of $\mu$-opioid receptor Gi/Go protein. This evidence suggests that ultra-low-dose naloxone alone might not only inhibit opioid receptor excitatory signaling but also re-establish the $\mu$-opioid receptor Gi/Go-protein coupling, and thus restore the balance between the excitatory and inhibitory signaling pathways and produce an antinociceptive effect of morphine. The detailed mechanisms of this hypothesis are under investigation.

In contrast to other opioids, McCormack et al (1998) had found that the antinociceptive effect of buprenophine was not blocked by the PTX treatment in rats (Womer et al, 1997). This raises the possibility that PTX treatment may not completely disrupt the signal transduction of all types of inhibitory Gi/Go-protein signaling, such as $\kappa$-opioid receptors. However, in our preliminary immunoprecipitation analysis, we found that PTX treatment completely disrupted all types of inhibitory $\mathrm{Gi}, \mathrm{Go}$, and $\mathrm{Gq}$ protein coupling to $\mu$-, $\kappa$-, and $\delta$-opioid receptors. Moreover, in our unpublished behavioral observation, administration of selective $\mu$-opioid receptor antagonist CTOP, but not $\kappa$-opioid receptor antagonist nor-BNI, blocked the effect of ultralow-dose naloxone on morphine's antinociception in PTXtreated rats. These results suggest that ultra-low-dose naloxone enhances the antinociceptive effect of morphine on PTX-induced thermal hyperalgesia in rats, probably via regulating $\mu$-opioid receptor G-protein signaling more than $\kappa$-opioid receptor signaling. The detailed mechanisms of ultra-low-dose naloxone on the regulation of opioid receptor G-protein signaling need further investigation.

In conclusion, ultra-low-dose naloxone pretreatment restores the antinociceptive effect of morphine, which is associated with a reduction in EAA concentrations in the CSF dialysate of PTX-treated rats. The mechanisms involve the inhibition of protein expression of pro-inflammatory cytokines IL- $1 \beta$ and IL- 6 and mRNA expression of TNF- $\alpha$,
IL-1 $\beta$, and IL-6 in spinal cord microglial cells. The upregulation of the expression of GTs, which, in turn, increases the uptake of extracellular L-glutamate into glial cells, subsequently stimulates GS expression, which further reduces neuronal excitotoxicity and neuroplasticity. On the basis of the results of our present study, we suggest that ultra-low-dose naloxone may be a useful analgesic adjuvant in patients suffering from neuropathic pain. It may be part of the reason why ultra-low-dose naloxone has been used in the treatment of chronic neuropathic pain syndromes via systemic administration in clinical conditions. The systemic effect and mechanisms of the actions of ultra-low-dose naloxone in neuropathic pain development require further investigation.

\section{ACKNOWLEDGEMENTS}

This study was supported by grants from the National Health Research Institutes, Taiwan (NHRI-EX96-9401NP) and the Chi-Mei Medical Center (95-CMNDMC02). This study was performed at the Nociception Signal Transduction Laboratory, Department of Anesthesiology, Tri-service General Hospital and National Defense Medical Center.

\section{DISCLOSURE/CONFLICT OF INTEREST}

We declare that, except for income received from their primary employer, no financial support or compensation has been received from any individual or corporate entity over the past three years for research or professional service and there are no personal financial holdings that could be perceived as constituting a potential conflict of interest.

\section{REFERENCES}

Campbell JN, Meyer RA (2006). Mechanisms of neuropathic pain. Neuron 52: 77-92.

Chen GG, Chalazonitis A, Shen KF, Crain SM (1988). Inhibitor of cyclic AMP-dependent protein kinase blocks opioid-induced prolongation of the action potential of mouse sensory ganglion neurons in dissociated cell cultures. Brain Res 462: 372-377.

Crain SM, Shen KF (1995). Ultra-low concentrations of naloxone selectively antagonize excitatory effects of morphine on sensory neurons, thereby increasing its antinociceptive potency and attenuating tolerance/dependence during chronic cotreatment. Proc Natl Acad Sci USA 92: 10540-10544.

Danbolt NC (2001). Glutamate uptake. Prog Neurobiol 65: 1-105.

Gilron I, Bailey JM, Tu D, Holden RR, Weaver DF, Houlden RL (2005). Morphine, gabapentin, or their combination for neuropathic pain. N Engl J Med 352: 1324-1334.

Grossman ML, Basbaum AI, Fields HL (1982). Afferent and efferent connections of the rat tail flick reflex (a model used to analyze pain control mechanisms). J Comp Neurol 206: 9-16.

Hardin-Pouzet H, Krakowski M, Bourbonniere L, Didier-Bazes M, Tran E, Owens T (1997). Glutamate metabolism is downregulated in astrocytes during experimental allergic encephalomyelitis. Glia 20: 79-85.

Kohno T, Ji RR, Ito N, Allchorne AJ, Befort K, Karchewski LA et al (2005). Peripheral axonal injury results in reduced mu opioid receptor pre- and post-synaptic action in the spinal cord. Pain 117: 77-87.

Li CY, Song YH, Higuera ES, Luo ZD (2004). Spinal dorsal horn calcium channel alpha2delta-1 subunit upregulation contributes 
to peripheral nerve injury-induced tactile allodynia. J Neurosci 24: 8494-8499.

Liu B, Hong JS (2003). Role of microglia in inflammation-mediated neurodegenerative diseases: mechanisms and strategies for therapeutic intervention. J Pharmacol Exp Ther 304: 1-7.

Liu B, Jiang JW, Wilson BC, Du L, Yang SN, Wang JY et al (2000). Systemic infusion of naloxone reduces degeneration of rat substantia nigral dopaminergic neurons induced by intranigral injection of lipopolysaccharide. J Pharmacol Exp Ther 295: 125-132.

Mao J, Sung B, Ji RR, Lim G (2002). Chronic morphine induces downregulation of spinal glutamate transporters: implications in morphine tolerance and abnormal pain sensitivity. J Neurosci 22: 8312-8323.

Marsala M, Malmberg AB, Yaksh TL (1995). The spinal loop dialysis catheter: characterization of use in the unanesthetized rat. J Neurosci Methods 62: 43-53.

McCormack K, Prather P, Chapleo C (1998). Some new insights into the effects of opioids in phasic and tonic nociceptive tests. Pain 78: 79-98.

Millan MJ, Colpaert FC (1990). Modification of nociception in a model of localized inflammatory pain by long-term administration of naloxone. Prog Clin Biol Res 328: 331-334.

Niederberger E, Schmidtko A, Rothstein JD, Geisslinger G, Tegeder I (2003). Modulation of spinal nociceptive processing through the glutamate transporter GLT-1. Neuroscience 116: 81-87.

Okada K, Yamashita U, Tsuji S (2005). Modulation of $\mathrm{Na}(+)$ dependent glutamate transporter of murine astrocytes by inflammatory mediators. J UOEH 27: 161-170.

Ozawa T, Nakagawa T, Shige K, Minami M, Satoh M (2001). Changes in the expression of glial glutamate transporters in the rat brain accompanied with morphine dependence and naloxone-precipitated withdrawal. Brain Res 905: 254-258.

Powell KJ, Abul-Husn NS, Jhamandas A, Olmstead MC, Beninger RJ, Jhamandas K (2002). Paradoxical effects of the opioid antagonist naltrexone on morphine analgesia, tolerance, and reward in rats. J Pharmacol Exp Ther 300: 588-596.

Raghavendra V, Rutkowski MD, DeLeo JA (2002). The role of spinal neuroimmune activation in morphine tolerance/hyperalgesia in neuropathic and sham-operated rats. J Neurosci 22: 9980-9989.

Robinson MB, Dowd LA (1997). Heterogeneity and functional properties of subtypes of sodium-dependent glutamate transporters in the mammalian central nervous system. $A d v$ Pharmacol 37: 69-115.

Shah S, Duttaroy A, Davis T, Yoburn BC (1994). Spinal and supraspinal effects of pertussis toxin on opioid analgesia. Pharmacol Biochem Behav 49: 773-776.

Shen KF, Crain SM (1997). Ultra-low doses of naltrexone or etorphine increase morphine's antinociceptive potency and attenuate tolerance/dependence in mice. Brain Res 757: 176-190.

Sitcheran R, Gupta P, Fisher PB, Baldwin AS (2005). Positive and negative regulation of EAAT2 by NF-kappaB: a role for N-myc in TNFalpha-controlled repression. EMBO J 24: 510-520.

Smart D, Lambert DG (1996). The stimulatory effects of opioids and their possible role in the development of tolerance. Trends Pharmacol Sci 17: 264-269.

Sudoh Y, Cahoon EE, Gerner P, Wang GK (2003). Tricyclic antidepressants as long-acting local anesthetics. Pain 103: 49-55.
Suarez I, Bodega G, Fernandez B (2002). Glutamine synthetase in brain: effect of ammonia. Neurochem Int 41: 123-142.

Sung CS, Wen ZH, Chang WK, Chan KH, Ho ST, Tsai SK et al (2005). Inhibition of p38 mitogen-activated protein kinase attenuates interleukin-1beta-induced thermal hyperalgesia and inducible nitric oxide synthase expression in the spinal cord. J Neurochem 94: 742-752.

Sung CS, Wen ZH, Chang WK, Ho ST, Tsai SK, Chang YC et al (2004). Intrathecal interleukin-1beta administration induces thermal hyperalgesia by activating inducible nitric oxide synthase expression in the rat spinal cord. Brain Res 1015: $145-153$.

Tai YH, Wang YH, Wang JJ, Tao PL, Tung CS, Wong CS (2006). Amitriptyline suppresses neuroinflammation and up-regulates glutamate transporters in morphine-tolerant rats. Pain 124: 77-86.

Tanaka Y, Otsuka T, Hotokebuchi T, Miyahara H, Nakashima H, Kuga $S$ et al (1996). Effect of IL-10 on collagen-induced arthritis in mice. Inflamm Res 45: 283-288.

Trujillo KA, Akil H (1991). Inhibition of morphine tolerance and dependence by the NMDA receptor antagonist MK-801. Science 251: 85-87.

Wang HY, Friedman E, Olmstead MC, Burns LH (2005). Ultra-lowdose naloxone suppresses opioid tolerance, dependence and associated changes in mu opioid receptor-G protein coupling and Gbetagamma signaling. Neuroscience 135: 247-261.

Watkins LR, Milligan ED, Maier SF (2003). Glial proinflammatory cytokines mediate exaggerated pain states: implications for clinical pain. Adv Exp Med Biol 521: 1-21.

Wen ZH, Guo YW, Chang YC, Wong CS (2003a). -2-Amino-5phosphonopentanoic acid inhibits intrathecal pertussis toxininduced thermal hyperalgesia and protein kinase Cgamma up-regulation. Brain Res 963: 1-7.

Wen ZH, Yang LC, Wang JJ, Chang YC, Hsing CH, Chen JY et al (2003b). Intrathecal pertussis toxin induces thermal hyperalgesia: involvement of excitatory and inhibitory amino acids. Neuroscience 116: 871-878.

Werner P, Pitt D, Raine CS (2001). Multiple sclerosis: altered glutamate homeostasis in lesions correlates with oligodendrocyte and axonal damage. Ann Neurol 50: 169-180.

Womer DE, DeLapp NW, Shannon HE (1997). Intrathecal pertussis toxin produces hyperalgesia and allodynia in mice. Pain 70: 223-228.

Wong CS, Chang YC, Yeh CC, Huang GS, Cherng CH (2002). Loss of intrathecal morphine analgesia in terminal cancer patients is associated with high levels of excitatory amino acids in the CSF. Can J Anaesth 49: 561-565.

Wong CS, Cherng CH, Tung CS (1998). Intrathecal administration of excitatory amino acid receptor antagonists or nitric oxide synthase inhibitor reduced autotomy behavior in rats. Anesth Analg 87: 605-608.

Wong CS, Su YF, Chang KJ, Watkins WD (1992). Intrathecal pertussis toxin treatment attenuates opioid antinociception and reduces high-affinity state of opioid receptors. Anesthesiology 77: 691-699.

Zelenaia OA, Robinson MB (2000). Degradation of glial glutamate transporter mRNAs is selectively blocked by inhibition of cellular transcription. J Neurochem 75: 2252-2258. 